\title{
Danksagung an die Reviewer im Jahr 2017
}

Online publiziert: 24. Januar 2018

๑) Springer-Verlag GmbH Deutschland, ein Teil von Springer Nature 2018

Manuskripte, die zur Veröffentlichung angeboten werden, werden durch Reviewer beurteilt. Diesen gilt ein besonderer Dank der Herausgeber. Am Reviewverfahren haben im Laufe des Jahres 2017 mitgewirkt:

Dr. Mareike Alscher, Humboldt-Universität zu Berlin;

Dr. Florian Bartholomae, Professur für Volkswirtschaftslehre, insbesondere Mikroökonomie und Wettbewerbspolitik, Universität der Bundeswehr München, Neubiberg;

Prof. Dr. Andreas Berkner, Regionaler Planungsverband Leipzig-Westsachsen, Leipzig;

Dr. Christine von Blanckenburg, nexus Institut für Kooperationsmanagement und interdisziplinäre Forschung, Berlin;

Prof. Dr. Hans Heinrich Blotevogel, Institut für Geographie und Regionalforschung, Universität Wien, Österreich;

Prof. Dr. Lorenz Blume, Universität Kassel;

Dr. Marco Bontje, Universiteit van Amsterdam, Niederlande;

Prof. Dr. Annegret Boos-Krüger, Hochschule für Angewandte Wissenschaften, München;

Dr. Barbara Breitschopf, Fraunhofer-Institut für Systemund Innovationsforschung, Karlsruhe;

Caroline Dangel-Vornbäumen, Deutscher LandFrauenverband, Berlin;
Prof. Simin Davoudi, School of Architecture, Planning and Landscape, Newcastle University, Newcastle, Großbritannien;

Prof. Dr. Benjamin Davy, Technische Universität Dortmund;

Dr. Jutta Deffner, Institut für sozial-ökologische Forschung, Frankfurt am Main;

Prof. Dr. Peter Dehne, Hochschule Neubrandenburg;

Dr. Fabian Dosch, Bundesinstitut für Bau-, Stadt- und Raumforschung im Bundesamt für Bauwesen und Raumordnung, Bonn;

Prof. Dr. Stefanie Dühr, Nijmegen School of Management, Radboud University, Nijmegen, Niederlande;

Klaus Einig, Ministerium für Inneres, ländliche Räume und Integration des Landes Schleswig-Holstein, Kiel;

Timo Fichtner, Amt für regionale Landesentwicklung Leine-Weser, Hildesheim;

Dr. Choni Flöther, Internationales Zentrum für Hochschulforschung, Universität Kassel;

Prof. Dr. Martin Franz, Institut für Geographie, Universität Osnabrück;

Prof. Dr. Klaus Friedrich, Institut für Geowissenschaften und Geographie, Martin-Luther-Universität Halle-Wittenberg;

Dr. Oliver Fritsch, University of Leeds, Großbritannien; 
Dr. Anita Grams, Institut für Raum- und Landschaftsentwicklung, Professur für Raumentwicklung, Eidgenössische Technische Hochschule Zürich, Schweiz;

Sören Gröbel, Institut für Siedlungs- und Wohnungswesen, Westfälische Wilhelms-Universität Münster;

Dr. Thomas Hartmann, Faculty of Geosciences, Utrecht University, Utrecht, Niederlande;

Prof. Dr. Dirk Heinrichs, Institut für Verkehrsforschung, Deutsches Zentrum für Luft- und Raumfahrt, Berlin;

Prof. Dr. Gerold Janssen, Leibniz-Institut für ökologische Raumentwicklung, Dresden;

Prof. Dr. Roger Keil, Faculty of Environmental Studies, York University, Toronto, Kanada;

Prof. Dr. Heiderose Kilper, Leibniz-Institut für Raumbezogene Sozialforschung, Erkner;

Heiko Kirchhain, EBS Universität für Wirtschaft und Recht, Wiesbaden;

Prof. Dr. Thomas Kistemann, Institut für Hygiene und Öffentliche Gesundheit, Rheinische Friedrich-Wilhelms-Universität Bonn;

Prof. Dr. Caroline Kramer, Institut für Geographie und Geoökologie, Karlsruher Institut für Technologie, Karlsruhe;

Prof. Dr. Martin Lendi, Eidgenössische Technische Hochschule Zürich, Schweiz;

Prof. Dr. Heike Liebmann, Brandenburgische Beratungsgesellschaft für Stadterneuerung und Modernisierung, Potsdam;

Prof. Dr.-Ing. Hans Joachim Linke, Institut für Geodäsie, Technische Universität Darmstadt;

Dr.-Ing. Stephan Löb, Niedersächsische Staatskanzlei, Hannover;

Dr.-Ing. Christian von Malottki, Institut Wohnen und Umwelt, Darmstadt;

Dr. Steffen Maretzke, Bundesinstitut für Bau-, Stadt- und Raumforschung im Bundesamt für Bauwesen und Raumordnung, Bonn;
Prof. Dr. Timothy Moss, Integrative Research Institute on Transformations of Human-Environment Systems, Humboldt-Universität zu Berlin;

Prof. Dr.-Ing. Hartmut Müller, Institut für Raumbezogene Informations- und Messtechnik, Hochschule Mainz;

Dr. Angelika Münter, ILS - Institut für Landes- und Stadtentwicklungsforschung, Dortmund;

Prof. Dr. Claudia Neu, Lehrstuhl für Soziologie Ländlicher Räume, Georg-August-Universität Göttingen;

Dr. Mario Neukirch, Institut für Sozialwissenschaften, Universität Stuttgart;

Matthias Piontek, Lehrstuhl für Unternehmensentwicklung, Innovation und wirtschaftlichen Wandel, Friedrich-SchillerUniversität Jena;

Dr. Marco Pütz, Eidgenössische Forschungsanstalt für Wald, Schnee und Landschaft, Birmensdorf, Schweiz;

Prof. Dr. Jürgen Rauh, Institut für Geographie und Geologie, Julius-Maximilians-Universität Würzburg;

Dr. Darja Reuschke, Department of Geography and Environment, University of Southampton, Großbritannien;

Dr. Klaus-Heiner Röhl, Institut der Deutschen Wirtschaft, Köln;

Dr. Mei-Ing Ruprecht, Landeshauptstadt Hannover;

Dr. Claus Schlömer, Bundesinstitut für Bau-, Stadt- und Raumforschung im Bundesamt für Bauwesen und Raumordnung, Bonn;

Dr. Frank Scholles, Institut für Umweltplanung, Gottfried Wilhelm Leibniz Universität Hannover;

Prof. Dr. Walter Seher, Institut für Raumplanung, Umweltplanung und Bodenordnung, Universität für Bodenkultur Wien, Österreich;

Dr. Mathias Siedhoff, Institut für Geographie, Technische Universität Dresden;

Prof. Dr. Annette Spellerberg, Fachgebiet Stadtsoziologie, Technische Universität Kaiserslautern;

Dr.-Ing. Martina Stepper, Lehrstuhl Stadtplanung, Technische Universität Kaiserslautern; 
Prof. Dr. Rolf Sternberg, Institut für Wirtschafts- und Kulturgeographie, Gottfried Wilhelm Leibniz Universität Hannover;

Jan Matthias Stielike, Bischofsheim (Rhön);

Dr. Thomas Thaler, Institut für Alpine Naturgefahren, Universität für Bodenkultur Wien, Österreich;

Martin Vaché, Institut Wohnen und Umwelt, Darmstadt;

Dr. Andrea Walter, Institut für Politikwissenschaft, Westfälische Wilhelms-Universität Münster;

Prof. Dr.-Ing. Alexandra Weitkamp, Professur für Landmanagement, Technische Universität Dresden;

Dr. Thomas Wieland, Karlsruher Institut für Technologie, Karlsruhe;

Prof. Dr. Rüdiger Wink, Fakultät Wirtschaftswissenschaften, Hochschule für Technik, Wirtschaft und Kultur, Leipzig;

Prof. Dr. Karsten Zimmermann, Technische Universität Dortmund; 